\title{
Cav-1 deletion impaired hematopoietic stem cell function
}

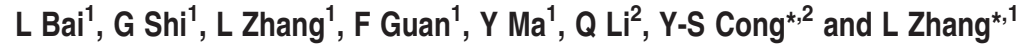

A tightly controlled balance between hematopoietic stem and progenitor cell compartments is required to maintain normal blood cell homeostasis throughout life, and this balance is regulated by intrinsic and extrinsic cellular factors. Cav-1 is a 22-kDa protein that is located in plasma membrane invaginations and is implicated in regulating neural stem cell and embryonic stem cell proliferation. However, the role of Cav-1 in hematopoietic stem cell (HSC) function is largely unknown. In this study, we used Cav-1 $1^{-I}$ mice to investigate the role of Cav-1 in HSCs function during aging. The results showed that Cav- $1^{-I-}$ mice displayed a decreased percentage of $B$ cells and an increased percentage of $M$ cells in the bone marrow and peripheral blood, and these changes were due to an increased number of HSCs. FACS analysis showed that the numbers of Lin ${ }^{-} \mathrm{Sca}^{+}{ }^{+} \mathrm{C}-\mathrm{kit}^{+}$ cells (LSKs), long-term HSCs (LT-HSCs), short-term HSCs and multipotent progenitors were increased in Cav-1 $1^{-l-}$ mice compared with Cav-1 ${ }^{+I+}$ mice, and this increase became more pronounced with aging. An in vitro clonogenic assay showed that LT-HSCs from Cav-1 ${ }^{-1-}$ mice had reduced ability to self-renew. Consistently, an in vivo competitive transplantation assay showed that Cav-1 ${ }^{-I-}$ mice failed to reconstitute hematopoiesis. Moreover, a Cav-1 deletion disrupted the quiescence of LSKs and promoted cell cycle progression through G2/M phase. In addition, we found that Cav-1 deletion impaired the ability of HSCs to differentiate into mature blood cells. Taken together, these data suggest that Cav-1-deficient cells impaired HSCs quiescence and induced environmental alterations, which limited HSCs self-renewal and function.

Cell Death and Disease (2014) 5, e1140; doi:10.1038/cddis.2014.105; published online 27 March 2014

Subject Category: Immunity

Adult tissue stem cells maintain and repair tissues by regulating an increased number of committed progenitor cells. However, the mechanisms that control adult stem cell homeostasis remain poorly understood, especially those involved during aging. A decline in tissue regenerative capacity and organ homeostasis is a major feature of aging that is associated with impaired stem cell function. ${ }^{1-3}$ Hematopoietic stem cells (HSCs) are well-characterized stem cells that have the highest self-renewal capacity and differentiation potential into hematopoietic progenitor and precursor cells. ${ }^{4}$ In aged mice, HSCs alter homing and mobilization properties, diminish the competitive repopulating ability and shift the lineage potential from lymphopoiesis toward myelopoiesis. ${ }^{5}$ However, how HSCs change with aging is not clear. It has been suggested that cell intrinsic and extrinsic factors can regulate stem cell function during aging. ${ }^{6,7}$ Cell intrinsic alterations, such as DNA damage, oxidative stress and senescence, are associated with and most likely contribute to HSC aging. ${ }^{8}$ The changes in environmental cues mediated via cell-cell interactions, cytokines and growth factors may also markedly contribute to age-dependent hematopoietic dysfunction. ${ }^{9,10}$

Caveolae are specialized invaginations of the plasma membrane that are implicated in a number of diverse cellular functions, such as signal transduction, lipid regulation, endocytosis and tumorigenesis. ${ }^{11}$ The major structural components of caveolae consist of the Cav (Cav-1, Cav-2 and Cav-3) and the Cavin (PTRF/Cavin-1, SDPR/Cavin-2, SRBC/Cavin-3 and MURC/Cavin-4) families. ${ }^{11-14}$ Cav-1 and Cav-2 are co-expressed and form a hetero-oligomeric complex in many cell types. ${ }^{15}$

Cav-1 is a major structural component of caveolae membranes and deletion of Cav-1 results in a nearly complete loss of caveolae. Young Cav-1 ${ }^{-1}$ mice are viable and fertile; however, at 27-65 weeks of age, these mice decline in viability. ${ }^{16}$ Loss of Cav-1 accelerates neurodegeneration and aging, ${ }^{17}$ and recent studies revealed that Cav-1 has a role in regulating neural stem cell (NSC) and embryonic stem cell (ESC) proliferation. Cav-1 increases NSC proliferation in the subventricular zone of the adult mouse brain, ${ }^{18}$ and Cav- 1 and

\footnotetext{
${ }^{1}$ Key Laboratory of Human Disease Comparative Medicine, Ministry of Health, Institute of Laboratory Animal Science, Chinese Academy of Medical Sciences and Comparative Medical Center, Peking Union Medical College, Beijing 100021, China and ${ }^{2}$ Institute of Aging Research, Hangzhou Normal University School of Medicine, Hangzhou 310036, China

${ }^{*}$ Corresponding author: Y-S Cong, Institute of Aging Research, Hangzhou Normal University School of Medicine, Hangzhou, China. Tel/Fax: +86 57128861693 ; E-mail: yscong@hznu.edu.cn

or L Zhang, Key Laboratory of Human Disease Comparative Medicine, Ministry of Health, Institute of Laboratory Animal Science, Chinese Academy of Medical Sciences and Comparative Medical Center, Peking Union Medical College, Chao Yang Strict, Pan Jia Yuan Nan Li No.5, Beijing 100021, China. Tel: +86 01087778442 ; Fax: +86 0106771 0812; E-mail: zhanglf@ cnilas.org

Keywords: Cav-1; HSC; quiescence

Abbreviations: Cav-1, caveolin-1; HSC, hematopoietic stem cell; FACS, fluorescence activated cell sorter; LSKs, Lin ${ }^{-}$Sca ${ }^{+}{ }^{+}$-kit ${ }^{+}$cells; LT-HSCs, long-term HSCs; ST-HSCs, short-term HSCs; MPPs, multipotent progenitors; ROS, reactive oxygen species; CLPs, common lymphoid progenitors; CMPs, common myeloid progenitors; GMPs, granulocyte-macrophage progenitors; MEPs, megakaryocyte-erythroid progenitors; BM, bone marrow; PB, peripheral blood

Received 27.9.13; revised 27.1.14; accepted 10.2.14; Edited by Y Shi
} 
integrin $\beta 1$ regulate ESC proliferation via p38 MAPK and FAK in high-glucose conditions. ${ }^{19}$ However, the role of Cav-1 in HSCs function has not been investigated. Additionally, Cav-1 has a critical role in the differentiation of monocytes into macrophages. ${ }^{20}$ And Cav-1 is regulated in B lymphocytes by LSP and involved in the development of $\mathrm{T}$-independent immune responses. The data suggest that Cav-1 may have an important role in the regulation of immune responses. ${ }^{21}$ Together, Cav-1 could play an important role in the regulation of HSCs function.

In this study, we used Cav-1 knockout mice to investigate the effect of Cav-1 on HSC self-renewal and proliferation during aging. We found that Cav-1-deficient mice exhibited impaired HSC/HPC development. In vitro and in vivo assays showed that the deletion of Cav-1-disrupted HSC function. In addition, HSCs from Cav-1 knockout mice increased reactive oxygen species (ROS) production and the percentage of cycling cells.

\section{Results}

Cav-1 knockout mice display a decreased number of $B$ lymphocytes. We first examined Cav-1 expression in the bone marrow (BM), spleen and thymus using RT-PCR and western blotting. Our results confirmed the absence of Cav-1 expression in Cav-1 $1^{-1-}$ mice (Figures 1a and b). We then measured the spleen weight and found that the spleen was larger in $\mathrm{Cav}-1^{-/}$mice compared with $\mathrm{Cav}-1^{+/+}$mice (Figure 1c). However, there were no apparent pathological changes between $\mathrm{Cav}-1^{+/+}$and $\mathrm{Cav}-1^{-/-}$mice, and there was no changes of the splenic white pulp (Supplementary Figures $2 a$ and $b)$. We used FACS analysis to determine the percentage of $\mathrm{B}$ cells $\left(\mathrm{B}^{2} 20^{+}\right)$, $\mathrm{T}$ cells $\left(\mathrm{CD} 3^{+}\right)$and $\mathrm{M}$ cells (monocytes and granulocytes, $\mathrm{CD}_{11 \mathrm{~B}}{ }^{+}$and $\mathrm{Gr}-1^{+}$) in the peripheral blood of 2-month (mo)-, 6-mo- and 12-mo-old mice. In $\mathrm{Cav}-1^{-1-}$ mice, the percentage of mature $B$ cells was reduced with aging (Figure 1d), and the percentage of $M$ cells was increased in 12-mo-old mice (Supplementary Figure 1a). However, no significant changes in the percentage of mature $\mathrm{T}$ cells (Figure 1d) between Cav-1 ${ }^{+/+}$and $\mathrm{Cav}-1^{-/-}$mice were observed. We also used FACS analysis to determine if any changes in the percentage of $B$ cells, T cells and $M$ cells in the BM, spleen and thymus occurred. In Cav-1 ${ }^{-1-}$ mice, the percentage of $\mathrm{B}$ cells in the $\mathrm{BM}$ was reduced with aging (Figure 1e), whereas the percentage of B cells in the spleen was not significantly decreased (Supplementary Figure 2c). The percentage of $M$ cells in the $B M$ was increased in Cav-1 $1^{-1}$ mice (Supplementary Figure 1a), but no significant changes in the percentage of $\mathrm{T}$ cells in the BM, thymus or spleen of $\mathrm{Cav}-1^{-/}$mice was evident (Supplementary Figures $1 \mathrm{~b}$ and $2 \mathrm{c}$ ). $\mathrm{Cav}-1^{-1-}$ mice had a reduced percentage of $B$ cells and an increased percentage of $M$ cells in the BM and PB, which was similar to what was observed during aging of WT mice. ${ }^{8,22}$

Cav-1 ${ }^{-I-}$ mice display hematopoietic stem/progenitor cell defects. Given that the percentage of $B$ cells decreased and the percentage of $\mathrm{M}$ cells increased in the BM and PB of Cav-1 $1^{-1}$ mice, we investigated whether this shift was related to an alteration in the stem/progenitor cell populations. We analyzed the LSK $\left(\mathrm{Lin}^{-} \mathrm{Sca} 1^{+} \mathrm{c}-\mathrm{kit}^{+}\right) \mathrm{BM}$ cell population of 2-mo- and 12-mo-old Cav-1 ${ }^{+/+}$and Cav-1 ${ }^{-1-}$ mice using FACS. The data showed that Cav-1 ${ }^{-1-}$ mice had a higher percentage of LSK cells within the total BM cell population that resulted in two- or threefold increases in the absolute number of LSKs (Figures $2 \mathrm{a}$ and $\mathrm{b}$ ). During aging, the differences between $\mathrm{Cav}-1^{+/+}$and $\mathrm{Cav}-1^{-I_{-}}$mice became more pronounced (Figure 2c). The numbers of LT-HSCs (long-term HSCs, $\mathrm{Lin}^{-} \mathrm{Sca}-1^{+} \mathrm{c}-\mathrm{Kit}^{+} \mathrm{CD} 34^{-}$ Flt- ${ }^{-}$), ST-HSCs (short-term HSCs, Lin ${ }^{-}$Sca- ${ }^{+}$ $\mathrm{C}-\mathrm{Kit}^{+} \mathrm{CD} 4^{+} \mathrm{Flt}^{-}{ }^{-}$) and MPPs (multipotent progenitors, $\mathrm{Lin}^{-} \mathrm{Sca}-1^{+} \mathrm{C}-\mathrm{Kit}^{+} \mathrm{Flt}-3^{+}$) were also detected using FACS a

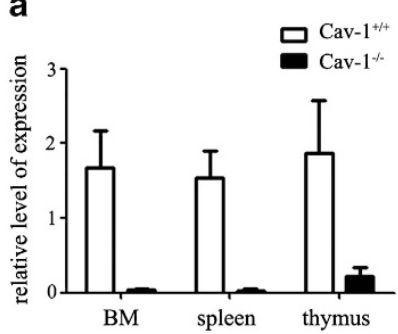

b

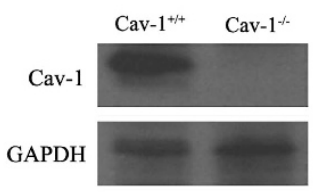

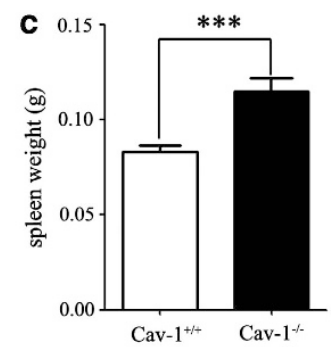
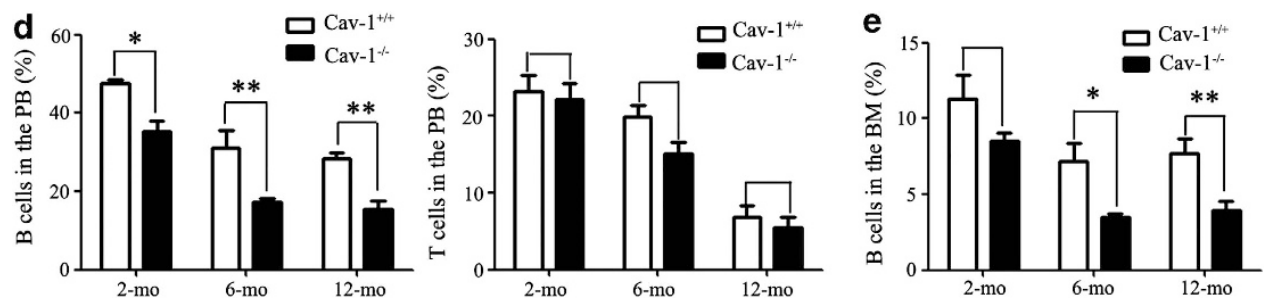

Figure 1 The percentage of B cells from the peripheral blood, BM and spleen was decreased in Cav-1 ${ }^{-1}$ mice. (a) Expression of the Cav-1 protein in the BM, spleen and thymus was detected using real-time PCR. (b) Expression of the Cav-1 protein in the BM was detected using western blotting. (c) The weight of the spleen from Cav- $1^{+1+}$ and Cav-1 ${ }^{-1-}$ mice. (d) FACS analysis of the percentage of B and T cells in the peripheral blood from 2-mo-, 6-mo- and 12-mo-old mice. (e) FACS analysis of the percentage of $B$ cells in the BM from 2-mo-, 6-mo- and 12-mo-old mice; $n=5$ mice per group. The experiment was repeated three times, and the results represent the mean \pm s.d. ${ }^{*} P<0.05 ;{ }^{* \star} P<0.01 ;{ }^{* \star *} P<0.001$. GAPDH expression was used for normalization 
a
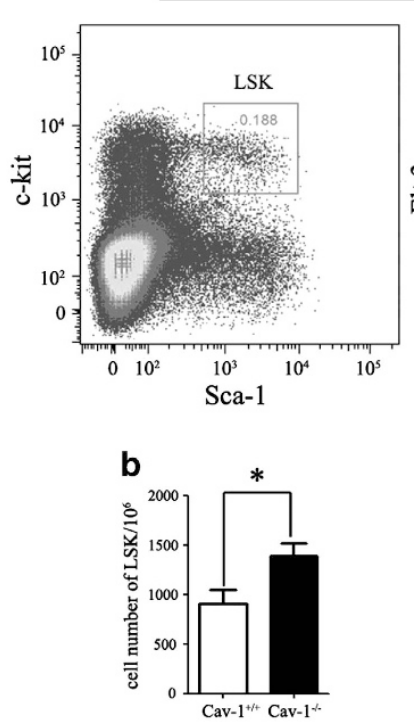

d

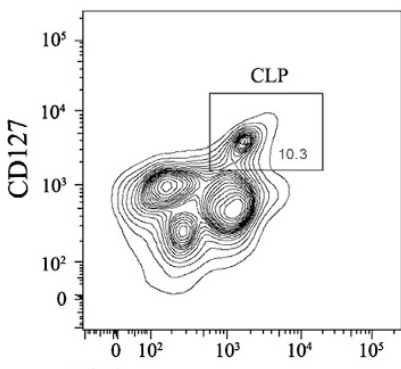

Cav-1+/+
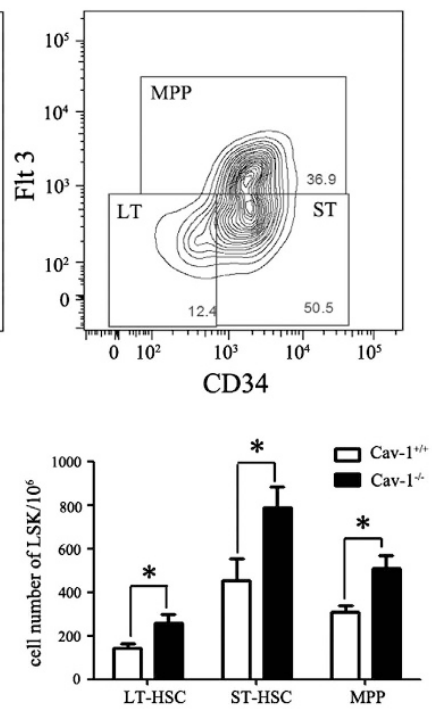

Cav-1\%

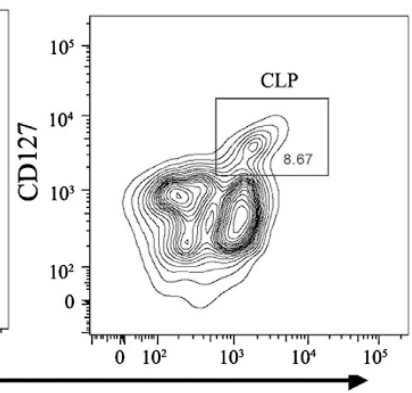

Cav-1
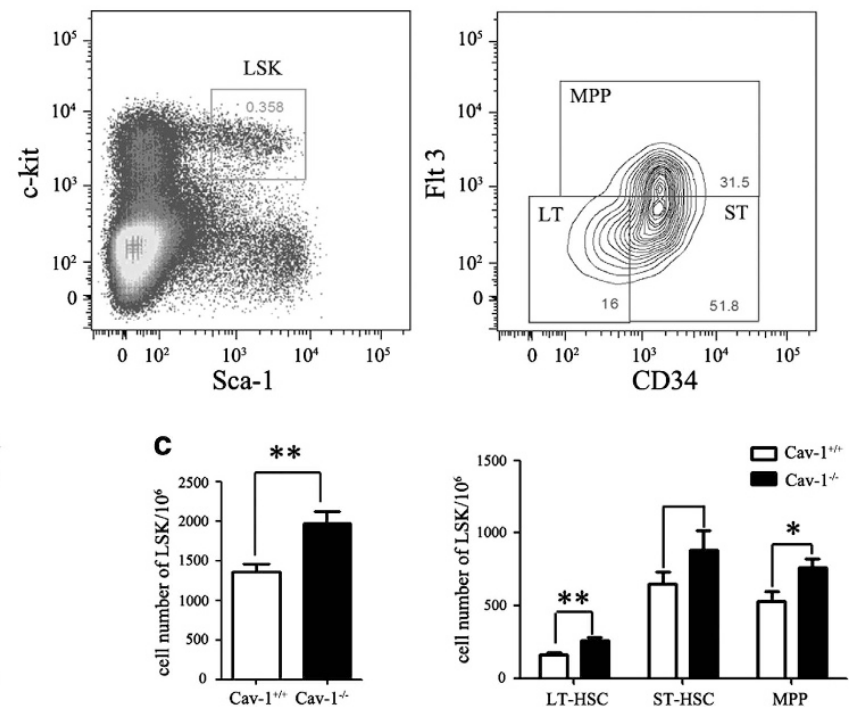

f

$\mathrm{Cav}_{-1}{ }^{+/+}$

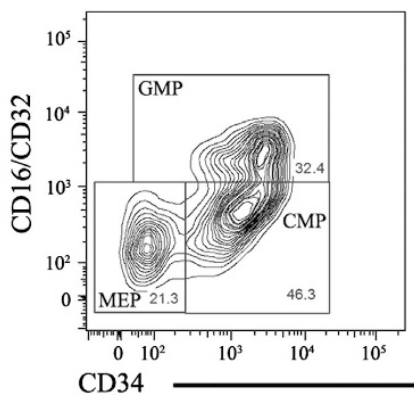

Cav-1 1
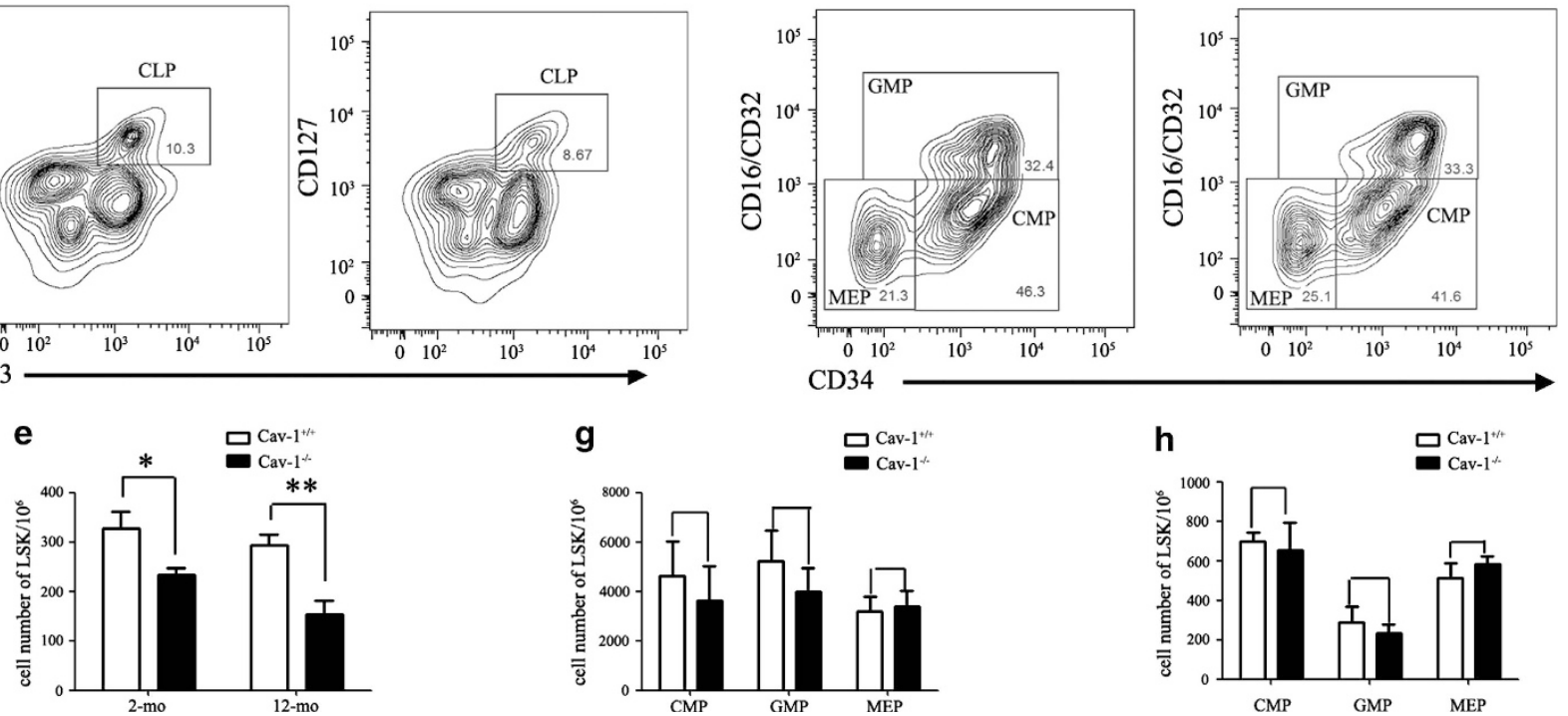

Figure 2 Hematopoietic stem/progenitor cell defects in Cav-1 ${ }^{-1}$ - mice. (a) Representative staining profiles for BM HSCs and progenitor populations. (b) Cell numbers of LSKs, LT-HSCs, ST-HSCs and MPPs in the BM from 2-mo- and 12-mo-old (c) mice. (d) Representative staining profiles for CLPs (Lin ${ }^{-}$Sca- $-{ }^{10 w}$ C-Kit $\left.{ }^{\text {low }} \mathrm{CD} 127^{+}\right)$in the BM. (e) Cell numbers of CLPs in the BM from 2-mo- and 12-mo-old mice. (f) Representative staining profiles for myeloid progenitors. $\mathrm{L}^{-} \mathrm{S}^{-} \mathrm{K}^{+}$cells were subdivided into CMPs

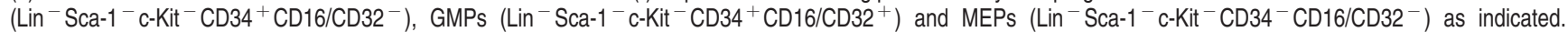
(g) Cell numbers of CMPs, GMPs and MEPs in the BM from 2-mo- and 12-mo-old (h) mice. $n=5$ mice per group. The experiment was repeated three times, and the results represent the mean \pm s.d. ${ }^{*} P<0.05 ;{ }^{*} P<0.01$

(Figure 2a). ${ }^{23}$ Cav-1 ${ }^{-1-}$ BM LSKs contained increased frequencies of LT-HSCs, but a marked increased frequency of ST-HSCs. When the measured frequencies were converted to absolute cell numbers, a marked increase of each population was noted (Figures $2 b$ and $c$ ). The increase in the numbers of HSCs and LT-HSCs in Cav-1 ${ }^{-1-}$ mice was more pronounced in 12-mo-old mice (Figure 2c).

Further differentiation of HSCs gives rise to common lymphoid progenitors (CLPs) and common myeloid progenitors (CMPs) in the BM. CLPs are derived from $\mathrm{c}-\mathrm{Kit}{ }^{\text {low }}$ Sca- $1^{\text {low }}$ Lin $^{-}$cells based on the expression of CD127. ${ }^{24,25}$ In $\mathrm{Cav}-1^{-/}$mice, a significant reduction in the frequency and absolute number of CLPs was observed (Figures $2 \mathrm{~d}$ and e).

CMPs are derived from $\mathrm{C}-\mathrm{Kit}^{+} \mathrm{Sca}-1^{-} \mathrm{Lin}^{-}$cells and can be further divided based on their CD34 and CD16/ CD32 expression into common myeloid progenitors (CMPs; CD34 ${ }^{+}$CD16/CD32 ${ }^{-}$), granulocyte-macrophage progenitors (GMPs; CD34 ${ }^{+}$CD16/CD32 ${ }^{+}$) and megakaryocyte-erythroid progenitors (MEPs; CD34- ${ }^{-}$CD16/CD32 ${ }^{-}$) (Figure 2f). In contrast, despite the reduced frequency of stem/progenitor cells, the frequency of myeloid progenitors (GMPs, EMPs and CMPs) in Cav-1 $1^{-1}$ mice was not significantly reduced compared with that of Cav-1 ${ }^{+/+}$mice (Figures $2 \mathrm{~g}$ and $\mathrm{h}$ ). 
Thus, the reduction of lymphoid populations, but not myeloid progenitor cell populations, and the increase of HSCs in Cav-1 ${ }^{-1}$ mice resembled the change in HSCs/HPCs and lymphoid populations that was previously described in Bmi-1 ${ }^{-1-24}$ and Ews ${ }^{-1-26}$ mice, all of which exhibit aginglike phenotypes.

Cav-1 ${ }^{-I}$ mice display disrupted HSC function. To investigate whether Cav-1 affects the proliferation and/or differentiation of HSCs and other cells, we performed in vitro clonogenic assays. We examined the clonogenic potential of LT-HSCs, BM and spleen cells derived from Cav-1 ${ }^{+/+}$ and Cav-1 ${ }^{-1-}$ mice. For the LT-HSCs, we sorted the Lin ${ }^{-}$ Sca- $1^{+} \mathrm{C}-\mathrm{Kit}^{+}{ }^{+} \mathrm{CD} 34^{-}{ }^{-} \mathrm{Flt}^{-}{ }^{-}$cells using FACS and then plated the cells in a methylcellulose-based medium. Fourteen days after plating, the colonies were counted using a microscope. Cells from Cav-1 ${ }^{-/}$mice displayed a reduced number of colonies compared with cells from Cav $-1^{+/+}$mice (Figure 3a). The decrease in colony size and number suggested a defect in the self-renewal of the LT-HSCs. We also sorted cells from the BM and spleen and found that the colony size and number were decreased in Cav-1 ${ }^{-1-}$ mice (Supplementary Figures $2 \mathrm{~d}$ and e), which suggested that the deletion of Cav-1 influences the proliferation and/or differentiation of BM and spleen cells.

We performed in vivo competitive repopulation experiments. BM cells from $\mathrm{Cav}-1^{+/+}$or $\mathrm{Cav}-1^{-1-}$ mice $\left(\mathrm{CD} 45.2^{+}\right)$were mixed at a ratio of $1: 1$ with competitor BM cells (CD45.1 ${ }^{+} 45.2^{+}$) and injected into lethally irradiated CD $45.1^{+}$-recipient mice. Flow cytometry analysis was used to determine the number of $\mathrm{CD} 45.2^{+}$-positive cells in the peripheral blood 2, 6, 10 and 14 weeks after transplantation. The results showed that cells derived from Cav-1 ${ }^{-1}$ mice exhibited a severe defect in the ability to reconstitute hematopoiesis in irradiated recipients (Figures $3 b$ and $c$ ).

To examine whether Cav-1 modulates the lineage of HSCs, at 14 weeks after transplantation, we used FACS to analyze the number of $B$ and $M$ cells in recipient mice. The results
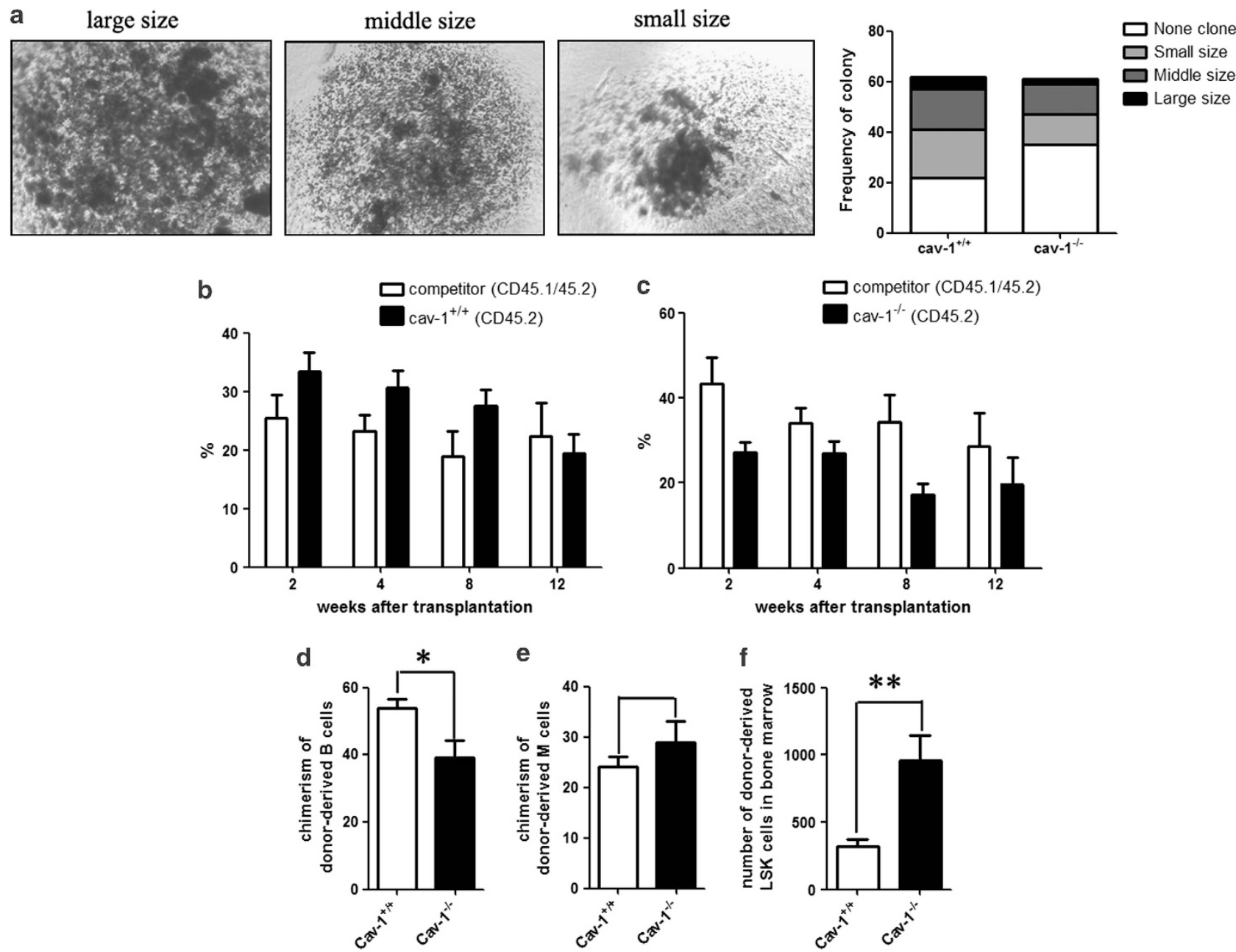

Figure 3 Loss of Cav-1 impairs the clonogenic ability and function of HSCs. (a) In vitro clonogenic potential of LT cells from Cav- $1^{+/+}$and Cav-1 ${ }^{-/-}$mice $(n=3)$. (b) The percentage of CD45.2+ $2^{+}$cells in the peripheral blood of irradiated recipient mice 2, 4, 8 and 12 weeks after transplantation with either Cav- $1^{+/+}$or Cav- $1^{-1-}$ cells (c) that were mixed at a 1:1 ratio with BM cells from competitors. (d) The percentage of donor-derived B and $\mathrm{M}$ cells (e) of either Cav- $1^{+/+}$or Cav-1 $1^{-1-}$ mice in the BM of recipient mice 12 weeks after transplantation. (f) The percentage of donor-derived LSK cells in the BM of recipient mice 12 weeks after transplantation. $n=9$ mice per group. The results represent the mean \pm s.d. ${ }^{*} P<0.05 ;{ }^{* *} P<0.01$ 
showed that in recipient mice transplanted with cells from Cav-1 ${ }^{-1-}$ mice, the chimerism of donor-derived B cells was decreased (Figure 3d), but that of donor-derived M cells was increased (Figure 3e). The number of LSKs was increased in recipient mice transplanted with cells from Cav-1 $1^{-/}$mice (Figure 3f). Thus, irradiated recipients of Cav-1-deficient cells failed to reconstitute hematopoiesis, and the function of LSKs was impacted.

Cav-1 $1^{-I-}$ cells induce a loss of HSC quiescence. The quiescence of HSCs has an indispensable role in the maintenance of the HSC pool throughout life, and disruption of HSC quiescence leads to the premature depletion of stem cells. The observed decrease in the frequency of HSCs in Cav-1 ${ }^{-1}$ mice raised the possibility that Cav-1 deletion compromised the quiescence of HSCs, which would lead to a reduction of the stem/progenitor cell size. To test this possibility, we characterized the cell cycle status of the stem/progenitor cells. We analyzed the proliferation of the LSK population using the cell marker Ki-67 and DNA content using the DNA dye 7-AAD. Cell cycle analysis showed that the percentage of cells in G0 phase was reduced, and more cells entered the $\mathrm{G} 1$ and $\mathrm{G} 2 / \mathrm{M}$ phases. This result indicated that the deletion of Cav-1 promotes stem cell exit from a quiescent state and entrance into G1 phase of the cell cycle, which is one of the common features found in HSCs from aged mice (Figures $4 a$ and $b$ ).

Oxidative stress is a critical determinant of HSC lifespan and self-renewal, and the loss of HSC quiescence frequently correlates with increased cellular ROS. ${ }^{27}$ Consistent with this, Cav-1 $1^{-1}$ HSCs displayed higher levels of ROS than Cav-1 ${ }^{+1+}$ HSCs. Thus, the increased ROS levels may induce the disrupted HSCs quiescence in $\mathrm{Cav}-1^{-/-}$mice (Figures $4 c$ and $d$ ).
Cav-1 deletion regulates several genes involved in cell cycle control and cellular senescence. Alterations in the HSCs function that is associated with Cav-1 deficiency can result in changes in the expression levels of key regulator genes. We sorted LSKs from Cav-1 ${ }^{+/+}$and Cav-1 ${ }^{-/-}$mice using FACS and used real-time PCR analysis to investigate several genes involved in the cell cycle, cellular senescence and $\mathrm{HSC}$ regulation.

No changes were observed in the expression levels of the cell cycle regulators p18 and p19 or in the Notch 1 pathway. However, Bmi-1, CXCR4 and p57 expression levels were downregulated in LSKs from Cav-1 ${ }^{-1-}$ mice, whereas the expression levels of the cellular senescence-associated proteins p16 and p21 were slightly upregulated (Figure 5). Taken together, these data indicate that several key regulators involved in the cell cycle, self-renewal, cellular senescence and adhesion may coordinate to regulate HSC function in $\mathrm{Cav}-1^{-1-}$ mice.

Cav-1 influences the environment of HSCs. Intrinsic and extrinsic cellular factors can regulate stem cell function during aging. To investigate whether Cav-1 alters the HSC environment, we transplanted BM cells from 2-mo-old RFP transgenic mice into cohorts of irradiated $\mathrm{Cav}-1^{+/+}$and Cav-1 $1^{-1}$ littermates. Four months after transplantation, the RFP + cell chimerism in the peripheral blood was not significantly different between the Cav- $1^{+/+}$- and Cav-1 ${ }^{-/-}$transplanted mice (Figure 6a). However, the percentage of $B$ and $\mathrm{T}$ cells in Cav-1 $1^{-1-}$-transplanted mice was decreased compared with the Cav-1 $1^{+/+}$-transplanted mice (Figure 6b). In the BM of Cav-1 $1^{-1-}$-transplanted mice, the frequency of $B$ cells was also significantly reduced (Figure 6c), whereas the number of LSK cells was still increased (Figure 6d). These data suggested that deletion of Cav-1 resulted in a
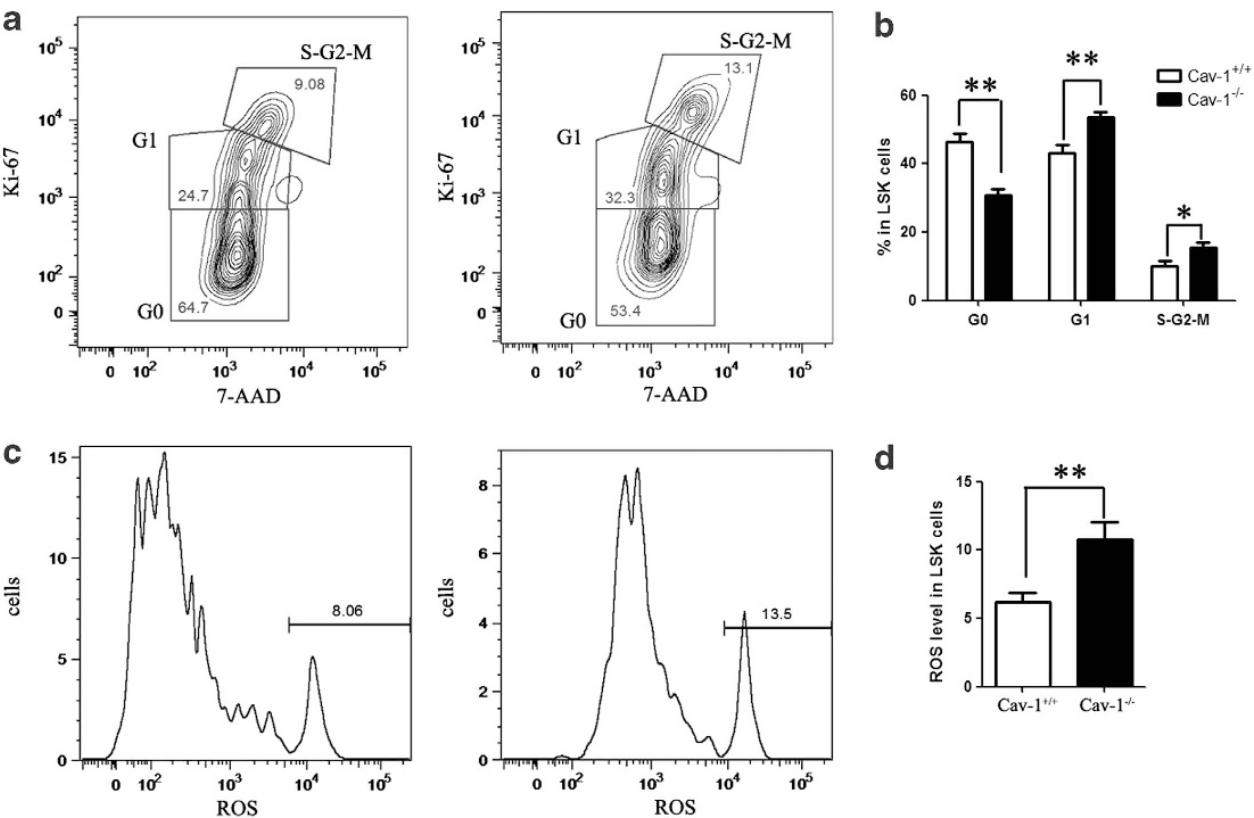

Figure 4 Deletion of Cav-1 induces a loss of HSC quiescence and accelerates cellular senescence. (a) FACS analysis of 7-AAD and Ki-67 staining of LSKs from Cav-1 ${ }^{+1+}$ or Cav-1 ${ }^{-1-}$ mice. (b) The percentage of cells in each phase of the cell cycle. (c) FACS analysis of LSKs from Cav- $1^{+1+}$ or Cav- $1^{-1-}$ mice using the ROS detection reagent. (d) The percentage of ROS-positive cells in the LSK population; $n=5$ mice per group. The results represent the mean \pm s.d. ${ }^{*} P<0.05 ;{ }^{*} P<0.01$ 


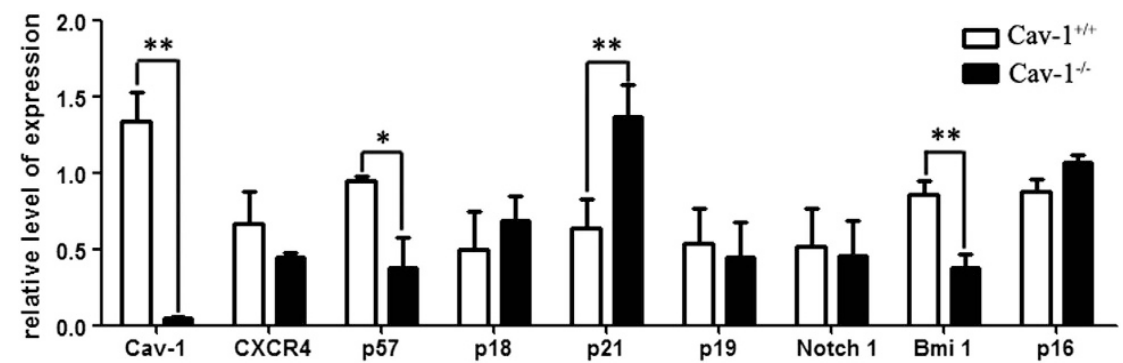

Figure 5 Cav-1 deletion altered the expression pattern of a number of transcription factors, cell cycle regulatory genes and cellular senescence-associated genes in LSKs. RT-PCR analysis of the gene expression of RNA isolated from sorted LSK cells from Cav- $1^{+1+}$ or Cav- $1^{-1-}$ mice. GAPDH expression was used for normalization. The experiment was repeated three times, and the results represent the mean \pm s.d. ${ }^{*} P<0.05 ;{ }^{* *} P<0.01$
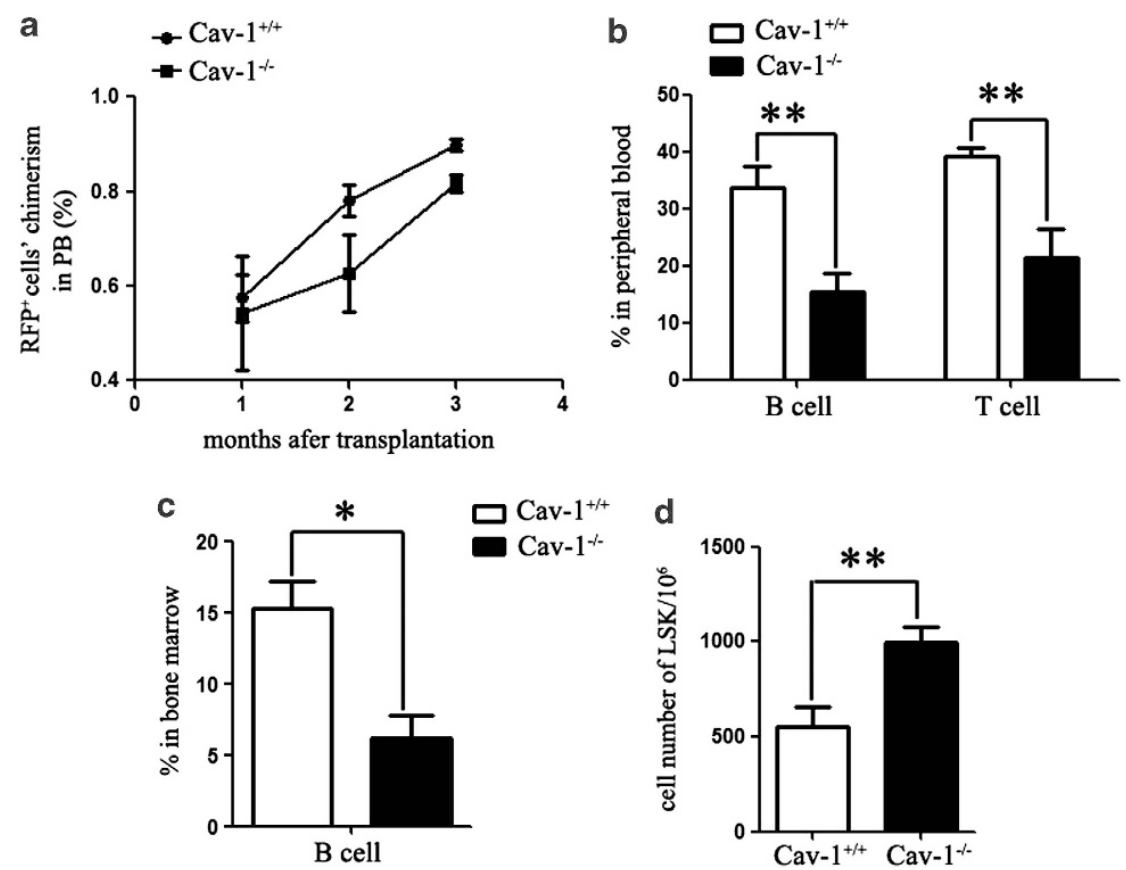

Figure 6 The Cav-1 $1^{-1-}$ environment induces the accumulation of LSK cells. (a) Chimerisms of RFP-derived peripheral blood cells were measured at the indicated time points after transplantation. (b) The percentage of RFP-derived B and T cells in the peripheral blood of Cav- $1^{+1+}$ or Cav-1 ${ }^{-1-}$ recipient mice 4 mo after transplantation. (c) The percentage of RFP-derived B cells in the BM of Cav- $1^{+1+}$ or Cav- $1^{-1}$ recipient mice 4 mo after transplantation. (d) Cell number of RFP-derived LSK cells in the BM of Cav-1 ${ }^{+1+}$ or Cav-1 $1^{-1-}$ recipient mice 4 mo after transplantation; $n=5$ mice per group. The experiment was repeated three times, and the results represent the mean \pm s.d. ${ }^{*} P<0.05 ;{ }^{* \star} P<0.01$

disruption of the HSC environment, which induced the proliferation but impaired the function of HSCs.

\section{Discussion}

In this study, we showed that Cav-1 has an important role in regulating HSC function. Cav-1 ${ }^{-1-}$ mice displayed a decreased percentage of $B$ cells and an increased percentage of $\mathrm{M}$ cells in the $\mathrm{BM}$ and $\mathrm{PB}$, and these changes were due to an increased number of HSCs. In vitro and in vivo assays showed that HSCs from Cav-1 ${ }^{-/-}$mice decreased the ability to self-renew and reconstitute hematopoiesis, and Cav-1 deletion disrupted the quiescence of LSKs and increased the level of produced ROS. However, Cav-1 deletion induced alterations in the HSC environment, increased HSC numbers and disrupted the ability of those HSCs to differentiate into mature blood cells.
It has been revealed that intrinsic and extrinsic cellular factors can contribute to a decline in stem cell function. Tight regulation of the cell cycle of HSCs is important for their function, and studies had implicated numerous factors in the regulation of the cell cycle status. However, how these factors interact to maintain the balance between HSCs quiescence and proliferation is an open question. ${ }^{28,29}$ Cav-1 inhibits the transcription of genes, such as cyclin D1, that are regulated during cell cycle progression. ${ }^{30}$ We showed that the cyclindependent kinase inhibitor p57, which was identified as a potential regulator of $\mathrm{HSC}$ quiescence, ${ }^{31}$ was downregulated in BM from Cav-1 ${ }^{-1-}$ mice. This change might disrupt the quiescence of HSC and promote cell cycle progression.

Additionally, Cav-1 modulated signal transduction by attracting signaling molecules to caveolae and regulating their activity. Many proteins, such as TGF $\beta$ R, PKC and the insulin receptor, ${ }^{6,15,32}$ that interacted with Cav-1 are involved 
in HSCs regulation. Thus, Cav-1 deletions may impact the interaction between cells and cytokines, which are growth factors that are associated with HSCs function.

The degree of oxidative damage has been found to increase with age in a variety of cells and tissues. Excess accumulation of ROS impacts cellular senescence, and the amount of ROS is a critical determinant of HSC lifespan and self-renewal. ROS are particularly deleterious to HSCs, especially as they age. ${ }^{33-35}$ Loss of LT-HSC quiescence frequently correlates with increased cellular ROS, which is negatively associated with HSC self-renewal. ${ }^{27} \mathrm{Cav}-1$ acts as a scaffolding protein that concentrates and functionally regulates signaling molecules, and the loss of Cav-1 was identified as a marker of the cancer-associated fibroblast phenotype. ${ }^{36}$ Different types of ROS have different effects on Cav-1 expression, cell migration and invasion. Superoxide anion and hydrogen peroxide downregulated Cav-1 expression and inhibited cell migration and invasion, whereas hydroxyl radicals upregulated Cav-1 expression and promoted cell migration and invasion. ${ }^{37}$ In this study, we found that Cav-1 deletion induced an increase in the level of produced ROS, and this increased production of ROS could be the reason for the loss of HSC function.

Taken together, these results indicate that deletion of Cav-1 induced alterations in the HSC environment and increased HSC numbers, but disrupted the ability of HSCs to differentiate into mature blood cells.

\section{Materials and Methods}

Animals. Cav- 1 knockout mice were obtained from the Jackson Laboratory (Sacramento, CA, USA) (strain name: STOCK Cav1 ${ }^{\text {tm1Mls }} / \mathrm{J}$ ), and the RFP transgenic mice were established in our laboratory. All of the mice had the C57BL/ $6 \mathrm{~J}$ genetic background, and all were maintained in a pathogen-free environment and fed a standard diet. The use of animals was approved by the Animal Care and Use Committee of the Institute of Laboratory Animal Science of Peking Union Medical College.

Flow cytometry. BM cells were flushed from tibias and femurs with PBS. The cells were filtered using a sterile nylon mesh and counted before staining with the indicated antibodies.

In most cases, the cells were stained using a biotin-conjugated anti-mouse CD4 (RM4-5), CD5 (53-7.3), CD8a (53-6.7), CD11b (M1/70), B220 (RA3-6B2), TER119 (TER-119) or Gr1 (RB6-8C5) antibody; and this was followed by staining with an APC-eFluor780-conjugated Streptavidin secondary antibody. The antibodies used for surface staining were as follows: PE CD71 (R17217), APC TER-119 (TER-119), PE-Cy7 B220 (RA3-6B2), APC IgM (II/41), FITC IgD (11-26), PE-Cy7 Sca-1 (D7), PE Flt3 (A2F10), FITC B220 (RA3-6B2), FITC CD34 (RAM34), PerCP-Cy5.5 CD127 (A7R34), PE CD16/CD32 (93), PerCP-Cy5.5 CD3e (145-2C11), PerCP-Cy5.5 CD45.2 (104), APC-eFluor780 CD11b, PE CD45.1 (A20) and APC CD117 (ACK2). All antibodies were obtained from eBioscience (San Diego, CA, USA).

Cell cycle analysis. For cell cycle analysis, all isolated BM cells were stained with stem cell surface markers (Lineage, Sca-1, c-kit), fixed, permeabilized (BD Bioscience, San Jose, CA, USA) and stained with a FITC Ki-67 antibody and 7-AAD. Data acquisition was performed using a FACSAria I cell sorter (Becton-Dickinson, Franklin Lakes, NJ, USA), and the acquired data were analyzed using FlowJo software (Treestar, Ashland, OR, USA).

Determination of ROS production. Cells were incubated with DCFH-DA (Beyotime Company, Hangzhou, China) at $37^{\circ} \mathrm{C}$ for $20 \mathrm{~min}$. DCFH-DA passively diffused into the cells and was deacetylated by esterases to form nonfluorescent $2^{\prime}, 7^{\prime}$-dichlorofluorescein (DCFH). The amount of emitted fluorescence correlated with the quantity of ROS in the cell. Data acquisition was performed using a
FACSAria I cell sorter (Becton-Dickinson), and the acquired data were analyzed using FlowJo software.

Competitive transplantation. In the competitive transplantation assays, BM cells $\left(2 \times 10^{6}\right)$ from 2-mo-old competitor $\left(\mathrm{CD} 45.1^{+} 45.2^{+}\right)$mice were mixed with CD45.2 ${ }^{+}$donor BM cells in a $1: 1$ ratio and transplanted into CD45.1 $1^{+}$ lethally irradiated host mice. The donor-derived chimerisms in the peripheral blood and BM were determined using FACS analysis 2, 6, 10 and 14 weeks after transplantation

Real-time PCR. Total RNA was extracted from cells using TRlzol reagent (Invitrogen, Carlsbad, CA, USA) according to the manufacturer's instructions. The genes of interest were amplified from DNase I-treated, total RNAs using M-MLV Reverse Transcriptase (Promega, Madison, WI, USA) and polydT primers. The primers that were used for PCR were as follows: Cav-1 (5'-GACCCCAAGCATCT CAACGAC- $3^{\prime}$ and $5^{\prime}$-GGATCGCAGAAGGTATGGACG- $3^{\prime}, \quad \mathrm{Tm}=62^{\circ} \mathrm{C}, \quad 27$ cycles), c-myc (5'-CTGGATTTCCTTTGGGCGTT- $3^{\prime}$ and $5^{\prime}$-AATAGGGCTGTAC GGAGTCG-3', Tm $=61^{\circ} \mathrm{C}, 30$ cycles), CXCR4 (5'-ATGGAACCGATCAGTGT GAG- $3^{\prime}$ and $5^{\prime}$-AAGTAGATGGTGGGCAGGAA- $3^{\prime}, \mathrm{Tm}=62^{\circ} \mathrm{C}, 35$ cycles), p 18 (5'-GGGACCTAGAGCAACTTACT- $3^{\prime}$ and $5^{\prime}$-TGACAGCAAAACCAGTTCCA- $3^{\prime}$, $\mathrm{Tm}=61^{\circ} \mathrm{C}, 30$ cycles), p21 $1^{\text {waf } 1 / \mathrm{cip}^{1}}\left(5^{\prime}\right.$-TCCAGACATTCAGAGCCACA-3' and $5^{\prime}$-CGAAGAGACAACGGCACACT-3', Tm $=60^{\circ} \mathrm{C}, 30$ cycles), p19 (5'-ATGGGT CGCAGGTTCTTGGT- $3^{\prime}$ and $5^{\prime}$-GTAGTGGGGTCCTCGCAGTT- $3^{\prime}, \mathrm{Tm}=61^{\circ} \mathrm{C}$, 35 cycles), p27 (5'-TCTCAGGCAAACTCTGAGGAC-3' and $5^{\prime}$-TTCGGAGCTGT TTACGTCTGG- $3^{\prime}, \mathrm{Tm}=61^{\circ} \mathrm{C}, 30$ cycles), Notch 1 (5'-CAGCCACAGGAACTTA CCACTCCAG- $3^{\prime}$ and $5^{\prime}$-TAAATGCCTCTGGAATGTGGGTGAT- $3^{\prime}, \mathrm{Tm}=57^{\circ} \mathrm{C}$, 35 cycles), Bmi-1 (5'-AGCAGCAATGACTGTGATGCACTTGAG-3' and 5'-GCTC TCCAGCATTCGTCAGTCCATCCC- $3^{\prime}, \mathrm{Tm}=57^{\circ} \mathrm{C}, 35$ cycles), p53 (5'-CATGAA CCGCCGACCTATC- $3^{\prime}$ and $5^{\prime}$-TCCCGGAACATCTCGAGGC- $3^{\prime}, \mathrm{Tm}=62^{\circ} \mathrm{C}, 35$ cycles), p16 (5'-CGAACTCTTTCGGTCGTACCC-3' and $5^{\prime}$-CGAATCTGCACCG TAGTTGAGC- $3^{\prime}, \mathrm{Tm}=62^{\circ} \mathrm{C}, 35$ cycles), p5 $7^{\mathrm{kip} 2}$ (5'-AGGAGCAGGACGAGAAT CAA- $3^{\prime}$ and $5^{\prime}$-TTCTCCTGCGCAGTTCTCTT- $3^{\prime}, \mathrm{Tm}=61^{\circ} \mathrm{C}, 30$ cycles), GAPDH (5'-GAGCGAGACCCCACTAACAT-3' and $5^{\prime}$-TTCACACCCATCACAAACAT- $3^{\prime}$, $\mathrm{Tm}=60^{\circ} \mathrm{C}, 25$ cycles). Real-time PCR using SYBR Premix Ex Taq II (TaKaRa, Shiga, Japan) was carried out using the $\mathrm{ABI}$ StepOne detection system (Applied Biosystems, Foster City, CA, USA).

Western blotting. For western blots, cell lysates were prepared in RIPA buffer ( $50 \mathrm{~mm}$ Tris-Cl (pH 8.0), $100 \mathrm{~mm} \mathrm{NaCl}, 0.1 \%$ SDS, $0.5 \%$ sodium deoxycholate, $1 \%$ NP-40) with a protease inhibitor cocktail (Roche, Reutlingen, Germany). Total protein concentrations were measured using a BCA kit, and immunoblotting of the clarified cell extracts was performed using $12 \%$ SDS-polyacrylamide gels. The proteins were transferred to nitrocellulose membranes (Millipore, Darmstadt, Germany) and incubated at $4^{\circ} \mathrm{C}$ overnight with the Cav-1 antibody (BD). An HRP-conjugated anti-rabbit secondary antibody was used for detection with a chemiluminescent detection system (Santa Cruz Biotechnology, Santa Cruz, CA, USA)

Clonogenic assays. Cells were plated in a methylcellulose-based medium (R\&D, HSC007). For LT-HSCs, we used 96-well cell culture plates (5 cells/well), with each sample added to 10 wells. For BM and spleen cells, we used 24-well cell culture plate $\left(10^{4} \mathrm{cells} /\right.$ well $)$, with each sample added to six wells. Two weeks after plating, the colonies were counted using a microscope.

Statistics. Data were analyzed using Microsoft Excel and GraphPad Prism software (GraphPad Software, San Diego, CA, USA). Differences were considered to be significant at $P<0.05$, as analyzed by Student's $t$-test. Error bars represent S.D. in all figures.

\section{Conflict of Interest}

The authors declare no conflict of interest.

Acknowledgements. This work was supported by the National Science Foundation of China (Grant No: 81200256, 31171320), Beijing Natural Science Foundation (Grant No.7132161) and the National Key Technology Research and Development Program of the Ministry of Science and Technology of China (2012BA139B02). 
1. Min $\mathrm{H}$, Montecino-Rodriguez $\mathrm{E}$, Dorshkind $\mathrm{K}$. Effects of aging on early $\mathrm{B}$ - and T-cell development. Immunol Rev 2005; 205: 7-17.

2. Ju Z, Jiang $\mathrm{H}$, Jaworski M, Rathinam $\mathrm{C}$, Gompf $\mathrm{A}$, Klein $\mathrm{C}$ et al. Telomere dysfunction induces environmental alterations limiting hematopoietic stem cell function and engraftment. Nat Med 2007; 13: 742-747.

3. Piccin D, Morshead CM. Potential and pitfalls of stem cell therapy in old age. Dis Model Mech 2010; 3: 421-425.

4. Orkin SH, Zon LI. Hematopoiesis: an evolving paradigm for stem cell biology. Cell 2008; 132: $631-644$.

5. Van Zant G, Liang Y. Concise review: hematopoietic stem cell aging, life span, and transplantation. Stem Cells Transl Med 2012; 1: 651-657.

6. Blank U, Karlsson G, Karlsson S. Signaling pathways governing stem-cell fate. Blood 2008; 111: 492-503.

7. Zon LI. Intrinsic and extrinsic control of haematopoietic stem-cell self-renewal. Nature 2008; 453: 306-313.

8. Rossi DJ, Bryder D, Zahn JM, Ahlenius H, Sonu R, Wagers AJ et al. Cell intrinsic alterations underlie hematopoietic stem cell aging. Proc Natl Acad Sci USA 2005; 102: 9194-9199.

9. Jasper H, Kennedy BK. Niche science: the aging stem cell. Cell Cycle 2012; 11: 2959-2960.

10. Wagner W, Horn P, Bork S, Ho AD. Aging of hematopoietic stem cells is regulated by the stem cell niche. Exp Gerontol 2008; 43: 974-980.

11. Parton RG, Simons K. The multiple faces of caveolae. Nat Rev Mol Cell Biol2007; 8: 185-194.

12. Hill MM, Bastiani M, Luetterforst R, Kirkham M, Kirkham A, Nixon SJ et al. PTRF-Cavin, a conserved cytoplasmic protein required for caveola formation and function. Cell 2008; 132 113-124.

13. Nabi IR. Cavin fever: regulating caveolae. Nat Cell Biol 2009; 11: 789-791.

14. Briand N, Dugail I, Le Lay S. Cavin proteins: New players in the caveolae field. Biochimie 2011; 93: 71-77.

15. Liu P, Rudick M, Anderson RG. Multiple functions of caveolin-1. J Biol Chem 2002; 277: 41295-41298.

16. Park DS, Cohen AW, Frank PG, Razani B, Lee H, Williams TM et al. Caveolin-1 null $(-/-)$ mice show dramatic reductions in life span. Biochemistry 2003; 42 : 15124-15131.

17. Head BP, Peart JN, Panneerselvam M, Yokoyama T, Pearn ML, Niesman IR et al. Loss of caveolin-1 accelerates neurodegeneration and aging. PLOS One 2010; 5: e15697.

18. Li Y, Luo J, Lau WM, Zheng G, Fu S, Wang TT et al. Caveolin-1 plays a crucial role in inhibiting neuronal differentiation of neural stem/progenitor cells via VEGF signalingdependent pathway. PLoS One 2011; 6: e22901.

19. Lee SH, Lee YJ, Park SW, Kim HS, Han HJ. Caveolin-1 and integrin beta1 regulate embryonic stem cell proliferation via p38 MAPK and FAK in high glucose. J Cell Physiol 2011; 226: 1850-1859.

20. Fu Y, Moore XL, Lee MK, Fernandez-Rojo MA, Parat MO, Parton RG et al. Caveolin-1 plays a critical role in the differentiation of monocytes into macrophages. Arterioscler Thromb Vasc Biol 2012; 32: e117-e125.

21. Medina FA, Williams TM, Sotgia F, Tanowitz HB, Lisanti MP. A novel role for caveolin-1 in $B$ lymphocyte function and the development of thymus-independent immune responses. Cell Cycle 2006; 5: 1865-1871
22. Guerrettaz LM, Johnson SA, Cambier JC. Acquired hematopoietic stem cell defects determine B-cell repertoire changes associated with aging. Proc Natl Acad Sci USA 2008; 105: 11898-11902.

23. Bai L, Shi G, Zhang X, Dong W, Zhang L. Transgenic expression of BRCA1 disturbs hematopoietic stem and progenitor cells quiescence and function. Exp Cell Res 2013; 319: 2739-2746.

24. Park IK, Qian D, Kiel M, Becker MW, Pihalja M, Weissman IL et al. Bmi-1 is required for maintenance of adult self-renewing haematopoietic stem cells. Nature 2003; 423 : 302-305.

25. Kondo M, Weissman IL, Akashi K. Identification of clonogenic common lymphoid progenitors in mouse bone marrow. Cell 1997; 91: 661-672.

26. Cho J, Shen $\mathrm{H}, \mathrm{Yu} \mathrm{H}$, Li H, Cheng T, Lee SB et al. Ewing sarcoma gene Ews regulates hematopoietic stem cell senescence. Blood 2011; 117: 1156-1166.

27. Pervaiz S, Taneja R, Ghaffari S. Oxidative stress regulation of stem and progenitor cells. Antioxid Redox Signal 2009; 11: 2777-2789.

28. Amrani YM, Gill J, Matevossian A, Alonzo ES, Yang C, Shieh JH et al. The Paf oncogene is essential for hematopoietic stem cell function and development. J Exp Med 2011; 208 1757-1765.

29. Ezoe S, Matsumura I, Satoh Y, Tanaka H, Kanakura Y. Cell cycle regulation in hematopoietic stem/progenitor cells. Cell Cycle 2004; 3: 314-318.

30. Hulit J, Bash T, Fu M, Galbiati F, Albanese C, Sage DR et al. The cyclin D1 gene is transcriptionally repressed by caveolin-1. J Biol Chem 2000; 275: 21203-21209.

31. Matsumoto A, Takeishi S, Kanie T, Susaki E, Onoyama I, Tateishi Y et al. p57 is required for quiescence and maintenance of adult hematopoietic stem cells. Cell Stem Cell 2011; 9 : 262-271.

32. Signer RA, Morrison SJ. Mechanisms that regulate stem cell aging and life span. Cell Stem Cell 2013; 12: 152-165.

33. Miyamoto K, Araki KY, Naka K, Arai F, Takubo K, Yamazaki S et al. Foxo3a is essential for maintenance of the hematopoietic stem cell pool. Cell Stem Cell 2007; 1: 101-112.

34. Ghaffari S. Oxidative stress in the regulation of normal and neoplastic hematopoiesis. Antioxid Redox Signal 2008; 10: 1923-1940.

35. Yalcin S, Marinkovic D, Mungamuri SK, Zhang X, Tong W, Sellers R et al. ROS-mediated amplification of $A K T / m T O R$ signalling pathway leads to myeloproliferative syndrome in Foxo3 ( - / - ) mice. EMBO J 2010; 29: 4118-4131.

36. Sotgia F, Martinez-Outschoorn UE, Howell A, Pestell RG, Pavlides S, Lisanti MP. Caveolin-1 and cancer metabolism in the tumor microenvironment: markers, models, and mechanisms. Annu Rev Pathol 2012; 7: 423-467.

37. Chanvorachote $P$, Chunhacha $P$. Caveolin-1 regulates endothelial adhesion of lung cancer cells via reactive oxygen species-dependent mechanism. PLoS One 2013; 8: e57466.

(c) (i) (2) Cell Death and Disease is an open-access journal (c) ${ }_{\text {BY }}$ SC SA licensed under a Creative Commons Attribution-NonCommercialShareAlike 3.0 Unported License. To view a copy of this license, visit http://creativecommons.org/licenses/by-nc-sa/3.0/

\section{Supplementary Information accompanies this paper on Cell Death and Disease website (http://www.nature.com/cddis)}

